\title{
Gradations in task engagement emerge from metacognitive priority control
}

\author{
Dominic Mussack (dominic.mussack@uni.lu) \\ University of Luxembourg; University of Minnesota, Twin Cities \\ Paul Schrater (schrater@umn.edu) \\ University of Minnesota, Twin Cities
}

\begin{abstract}
Engagement is a critical motivational factor that has broad effects on learning, productivity, performance, and even satisfaction and happiness. However, engagement can be impacted by a myriad of factors including the features of the task, the broader environment and the person's needs and biological states. Engagement has been difficult to characterize and measure, resulting in domain-specific conceptualizations and models that vary widely, creating a disconnected view of the overall phenomena of task engagement. Here we address this problem by developing an integrated metacognitive framework for understanding task engagement. We identify the key phenomenal characteristics of task engagement as integrating a graded effort for ongoing tasks with the temporal dynamics of task switching. We treat engagement as resulting from a unified meta-cognitive decision process that handles the gradient of engagement as resulting from a common priority calculation, the equivalent of an overall utility rate for ongoing tasks. Priority signals are computed relative to a set of available tasks and updated across time and changes in the environment. We propose a metacognitive controller makes decisions about both task switching (when to quit, next task) and cognitive resourcing (working memory, attention, etc) using the graded priority signals. By simultaneously choosing the task and allocating resources using the same graded signals, we capture the complex dependencies of engagement with task errors, performance, and time allocation.
\end{abstract}

Keywords: engagement; motivation; meta-cognition; scheduling; time allocation

\section{Introduction}

Consider the course of engagement involved in writing (see Figure 1). A writer might clear her schedule, makes sure she has a cup of coffee and good breakfast, and start the writing. However engagement is not instant. First, she spends time recalling or reading through what she previously wrote to remember much of her ideas, and then spending time sketching out what paragraphs to writes. Once she start producing new text her immersion still fluctuates. She might switch to other activities entirely due to externally prompted or selfinterruptions. A particular paragraph might be difficult to phrase so breaks from writing all together and checks Twitter. Or breakfast was not big enough, so she gets hungry and goes for a snack. Perhaps she also has planned interruptions; after an hour go refresh her coffee, think through what to do next, and sit back down.

We engage in a host of different tasks and activities over time - writing a paper, playing a game, eating lunch with friends, or sleeping. We switch from one activity to another, and our absorption in these activities fluctuates. Some of this fluctuation and switching is driven by relatively clear external constraints (e.g., work deadlines) or internal biological drives (e.g., hunger). However sometimes disengagement

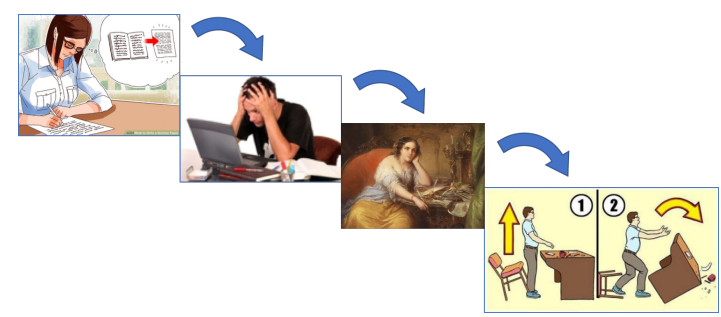

Figure 1: Engagement in writing can often move from flow, to split attention, to mind wandering, to disengagement

is inexplicable; a person might begin work on a paper, but their progress slows, their mind wanders, and finally decide to switch away to something else. How do we understand what determines this change in engagement?

Engagement has emerged as a key concept across a broad array of domains, including learning (D'Mello, Dieterle, \& Duckworth, 2017), education (Sinatra, Heddy, \& Lombardi, 2015; Reschly \& Christenson, 2012), the design of games (Boyle, Connolly, Hainey, \& Boyle, 2012) and user interfaces (Bouvier, Lavou, \& Sehaba, 2014). While it's widely recognized that engagement is not a unitary construct either within or between these domains, there are several general shared characteristics common across all characterizations. Engagement is viewed as a connection between a person and a task, goal, or domain that results in allocation of time and energy towards the foci of the connection. While some uses of engagement refer to processes on a timescale of weeks, our interest is in cognitive engagement at the level of tasks.

Task engagement refers to a gradient of immersion in a task; from experiencing flow (Csikszentmihalyi \& Lefevre, 1989) to partially engaged multitasking (Rosen, Carrier, \& Cheever, 2013; Kane, Kwapil, Mcvay, \& Myin-germeys, 2007) to full task disengagement. This gradient corresponds to a matching gradient of resource allocation. These task resources include energetic, cognitive, and attentional resources, but also critically time resources. Disengagement represents a distinct problem of time allocation, when to stop, and we can gain insight into how motivation relates to engagement by separately investigating this scheduling problem. Previous views on engagement do not fully integrate these phenomena. In this paper we clarify the phenomena of engagement, and propose a meta-cognitive architecture that sets the priority of tasks. Since priority is determining both resource and time allocation, this integrates seemingly disjoint components of engagement. 


\section{Phenomenon of task engagement}

Our writing example above emphasizes many of the critical components of engagement: a task or activity to be engaged in, while engaged the activity at least subjectively appears easier, and as disengagement happens it is more difficult to focus. There are nonintentional (e.g., boredom or hunger) intrusions into engagement, which can be deliberately worked around through shaping the environment or planning for intrusions. And, importantly, disengagement results in engagement in another separate task or activity. We can visualize engagement as both a discrete or continuous process (see Figure 2), as immersion towards some particular goal and disjoint task switching.

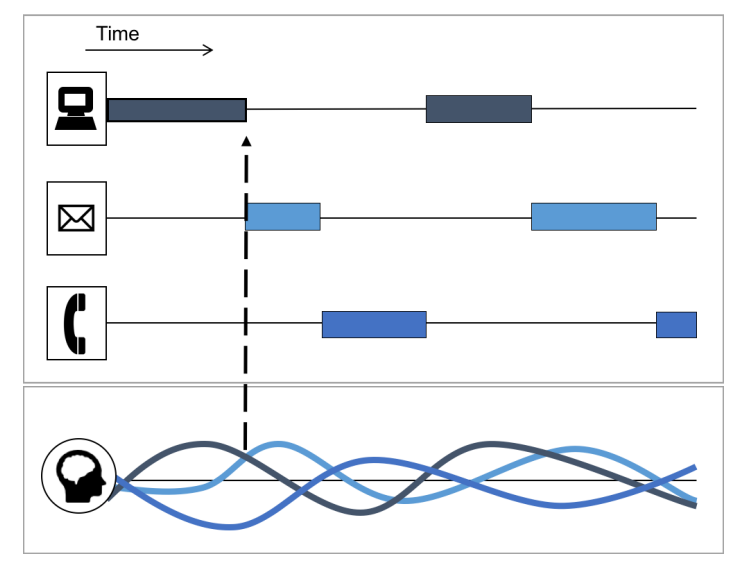

Figure 2: (top) A Gantt chart, representing what task a person is engaged with at what time. (bottom) Continuous engagement in each of the three tasks. This motivational engagement drives the more discrete task switching above.

Another prototypical example of engagement is video games. While immersed in a Mario game, a player might spend time trying to beat a particularly difficult level. She might fail multiple times, and get emotionally frustrated, but stay absorbed. Eventually, through effort and skill, she reaches a new level. Later she might quit despite not finishing or encountering any particular challenge; she spontaneously quits. The player's engagement, their immersion and time spent in a game, can fluctuate due to events in the game (e.g., beating a level) and out of the game (e.g., getting tired). However within game elements are often the focus of game developers and researchers.

Many people spontaneously engage in game play without external incentives, as attempts to 'gamify' education emphasize. Gamification tries to capture the aspects of games that promote engagement and apply that to schooling. The hope is that if incorporate into schooling game features that promote engagement, students might persist in spite of task failure, as people often do in games. Successful modification involves trying to isolate and translate the motivational impetus for gaming to educational domains (Ryan, Rigby, \& Przybylski, 2006). Here the divergent concepts and measures of en- gagement between pedagogy and game design form a serious impediment (Whitton \& Moseley, 2014).

Engagement in this paper refers to the result of cognitive resource allocation towards goals. Motivation is often considered the impetus or cause of engagement; motivation towards a task reflects the value associated with that goal (Dickinson $\&$ Balleine, n.d.) Neither engagement nor motivation are directly observable; they produce indirect outcomes in terms of what and how people perform different tasks. Motivation and engagement are latent variables, i.e., unobservable variables that are not directly measurable via objective methods. These latent variables have to be inferred in terms of their impact on task choice, time allocation, task performance, or through psychophysiology and neuroimaging methods.

Motivation and engagement are both distinct from subjective enjoyment and deliberate desire, however these appear to impact both at least distally. These distinctions are important when considering the causal relation between motivation and engagement. Rather than operationalizing engagement via a single measure, such as vigilance-related cerebral bloodflow (Matthews et al., 2010), this paper considers engagement as being caused by an integrated computation across tasks.

A task can be considered a distinct segment of behavior that is defined based on a particular goal (Newell \& Card, 1985). A focus on laboratory experimentation means that, in cognitive science, tasks are defined based upon explicit goals and instructions an experimenter presents to a participant. However beyond the lab tasks are not as well defined. In our examples, the Mario video game can provide a precise definition of tasks due to the programmatic specification of the game state and the clear success and failures. However, in our writing example, there is far more task ambiguity. Our focus is on tasks concerning more proximate goals at the time-course of within a particular day, rather than transitions between subtask motor activity or monthly or annual biological rhythms. This corresponds to the rational level of timescale as distinguished by (Newell \& Card, 1985), with a focus on distinct or mutually exclusive goals humans can switch between. Therefore our interest in motivated engagement also concerns daily fluctuations in motivation.

\section{Gradient of engagement}

While we can describe someone's engagement as all or none, engagement can better be described as a graded phenomenon (see Figure 3). Engagement can refer to a broad gradient of different levels of engagement, immersion, and absorption in a task or activity. People can engage in a task to varying degrees, including complete disengagement where people switch from one task to another. Psychological flow represents one extreme of 'optimal engagement' (Csikszentmihalyi \& Lefevre, 1989), where all available cognitive resources are allocated and a person is wholly immersed in a particular task. For the video game player, flow involves the external world disappearing, which might only reappear when boredom sets in. On the other had, off-task mind wandering, as in our writing example, represents a pas- 
sive disengagement from a task; although the writer is performing the task, they are allocating minimal resources without fully switching to a new task (Kane et al., 2007).

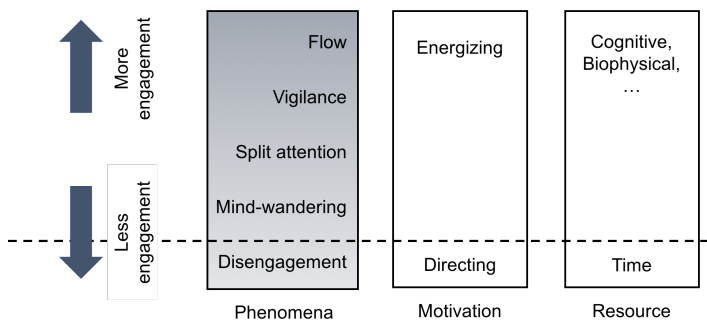

Figure 3: The gradient of engagement. Listed various engagement releated phenomena and where they lie - flow is full engagement while disengagement is none. The dotted line represents a bifurcation point in engagement, where someone moves from partial to complete disengagement. The motivational distinction between energizing and directing relate to these phenomena, and to the distinct resources.

This gradient in resource allocation parallels a similar gradient in ecological fear (Mobbs, Hagan, Dalgleish, Silston, \& Prevost, 2015). Animals have to deal with the cost of predation while still engaging in other activities, and so require a gradient of 'fearful' responses from partial vigilence to imminent defense (Stephens, Brown, \& Ydenberg, 2007). Much like engagement, vigilence is operationalized differently in different research contexts (e.g., across species). In some instances vigilence refers to time spent looking for predators exclusively, while in others it refers to split attention while performing other tasks. This 'parallel' versus 'serial' engagement distinction also exists, and motivation is relevant for either engagement process.

Within motivational theory a distinction is often made between the directing aspect of motivation and the energizing component (Niv, Joel, \& Dayan, 2006; Dickinson \& Balleine, n.d.). The directing component refers to motivations directed towards distinct tasks (e.g., eating vs sleep), while the energizing refers to the impetus or force applied to a task (e.g., how vigorously to eat). These different aspects of motivation likely relate to different parts of the gradient of engagement, and suggest that the decision problem of engagement might refer to related but distinct decisions.

\section{Factors that impact engagement}

Engagement is influences by various different features, including fatigue, deadlines, interest, temptations, and possible background activities. External features, most obviously extrinsic reward, impacts engagement given standard reinforcement (Schüll \& Library., 2012). Points in games have mixed success, and can act like rewards (Hoffman \& Nadelson, 2010). Internal drives also impact engagement, in that homeostatic states such as hunger or sleep will prompt engagement in relevant tasks (Niv et al., 2006), including changes in task selection and vigor. Similarly, intrinsic drives to engage in a task for its own sake (e.g., curiosity, mastery), rather than for an "extrinsic reward", also impacts engagement (Ryan et al., 2006).

Task expectation and task progress has also been shown to impact engagement (Kidd, Palmeri, \& Aslin, 2013; Carver, C. S., \& Scheier, 1998). Task deadlines can be seen to impact engagement in a similar way, by changing the likelihood of completion if the task is performed at a certain time (Hartonen \& Alava, 2013). Task progress provides information about the immediate likelihood of task completion, or goal satisfaction, and so could determine quitting during task engagement

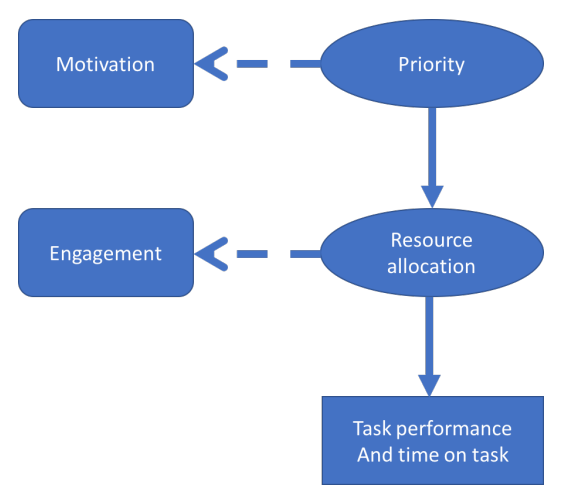

Figure 4: Goal priority determines resource allocation (time, cognitive resources) which can determine task performance along with goal satisfaction. However, priority and resource allocation are not directly observable and are instead latent variables. The results of the resource allocation, such as task performance and time on task, are observable. Here, we relate the experience of motivation towards a goal as the subjective reflection of the priority that goal has. Similarly, the experience of task engagement is the subjective reflection of various resource allocation (e.g., attentional). Generally, engagement follows motivation. However in some instances, such as externally enforced constraints on time on tasks, engagement and motivation can be disjoint. Similarly, engagement requires resources to allocate; if few resources exist (e.g., due to fatigue), then engagement might be low relative to motivation. Hence we might "desire to do something" separately of actually engagement.

More abstractly, overall environmental features can impact engagement, as in fear of predation (Mobbs et al., 2015). Engaging in one task induces an opportunity cost by not engaging in others, implying that quality of an environment can impact task time allocation by signaling the quality of alternative tasks. This is directly shown in optimal foraging theory, which indicates a tradeoff in foreground and background quality on time in task (Stephens et al., 2007). When the environmental quality is high, humans might spend little time in any task (they 'skim the fat' off of all tasks). Alternatively in a low quality environment humans might spend more time in each task (they stick to the 'oasis in a desert'). While the en- 
vironment can be literal as in ecology research, it can also be a more abstract 'task environment'. Many of these environmental features are reflected in how mood impacts engagement (Eldar, Rutledge, Dolan, \& Niv, 2016).

\section{Meta-cognitive control}

Engagement is the result of a meta-cognitive process, as the problem of engagement refers to what tasks to engage in and when rather than determining the low level actions that solve a given task (see figure 5). Engagement in a task says nothing about the problem that cognitive, perceptual, and motor resources are allocated to. Instead it concerns how these resources are allocated across tasks; engagement represents a decision to allocate resources to a task. People more engaged in a task allocate more resources, and those less engaged allocate less. However the types of resources can qualitatively change the nature of engagement, which might explain differences in how engagement has been defined across fields.

\section{Priority as jointly specifying resources, time, and task}

We propose that engagement is the result of a meta-cognitive priority control system (see Figure 5. Task-level control is achieved by different local tasks actors that control immediate behavior. A task queue retains the set of available tasks that are most likely switched between (based on a priority cutoff for low-priority tasks, i.e., "swim to the moon" is not a viable task). These task controllers vie for resources and immediate control based on a priority score that is computed by a metacognitive priority controller.

The meta-cognitive controller is similar to many actorcritic models (Sutton \& Barto, 1998), in that a meta-critic evaluates the overall priority across tasks, environments, and history, and produces weighted priority scores for a metaactor. This meta-actor then sets resources (which can be graded) and which task controller is currently active (i.e., the scheduling and time allocated towards each task controller). Each local task controller can also be an actor-critic network, allowing allocated resources that enable monitoring, learning, or updating based upon off-task information.

Since all meta-actions are produced by an overall priority score, this produces a covariance between resource and time allocation, and therefore a covariance between various engagement phenomena. We now discuss the various resource types including task resources e.g., attention and energy, as well as time and scheduling of tasks.

\section{Task resources}

When engaged in a task people allocate a set of resources towards that task. These resources include biophysical ones such as energetic expenditure during overt movement, but also cognitive and perceptual resources. While some mental processes occur automatically (e.g., object recognition), others are limited and must be allocated (e.g., working memory). The ability to maintain concentration, attention, or vigilance during a task, often despite task difficulty, can be attributed to either energetic or cognitive limits (Matthews et al., 2010) in the form of resources. Cognitive control (i.e., executive function) refers to the set of cognitive process that determines how these limited resources are allocated. The relationship between cognitive control and motivation, therefore, investigates how motivation can impact allocation of cognitive resources (Botvinick \& Braver, 2014). Cognitive control can be factored into distinct components such as inhibition, task switching, and working memory processing (Miyake et al., 2000), each of which might engage various resources that are either depleted or used.

Working memory is perhaps the most well-known limited cognitive resources; people can generally only keep in active thought a limited amount of information. These working memory resources store task-relevant information for a period of time as needed to complete the immediate task - for our writer they involve both what has been and what needs to be written. However, working memory can also be used for processing information that has nothing to do with the current focal task - 'Task Unrelated Thoughts' (TUTs) (Kane et al., 2007) such as what to make for dinner or whether I need to do laundry. Attentional resources are similar to working memory, in that they refer to a limited bandwidth of information that can be processed, but can be taken up by task irrelevant 'distractors'.

Task switching with respect to cognitive control refers to how people manipulate and switch mental 'task sets' or 'schema' - a set of cognitive resources, potential solutions, or policies that might solve a given task (Miyake et al., 2000). While the tasks used in many task switching experiments are very simplistic compared to our writing or game examples, they point to limits in multitasking based on a constrained set of resources. Research on task switching points to a general set of psychological difficulties and negative impact on performance demands due to multitasking (Carrier, Rosen, Cheever, \& Lim, 2015) — while what the specific resources are is still debated, it is important to emphasize their impact on task behavior and engagement. For our writer this can be clearly seen, as it takes time to actually engage these resources.

Interestingly, people display an inability to anticipate how task switches will reduce their performance on important tasks, even though both external and internal distractions are common (Rosen et al., 2013). For example, (Kessler, Shencar, \& Meiran, 2009) investigated whether people would switch given the associated performance costs. Importantly while subjects incurred a performance cost due to switching, they still decided to spontaneously switch tasks rather than keep to a single task ${ }^{1}$.

These self-interruptions are often explained as failures of

\footnotetext{
${ }^{1}$ While arguably not surprising, (Kessler et al., 2009) claim that this behavior is not predicted based on current theories of cognitive control. From p127, "The fact that no one has shown this phenomenon before and that those who studied 'voluntary switching' always took precautions to ensure that participants would switch tasks suggests that this phenomenon has not been predicted"
} 


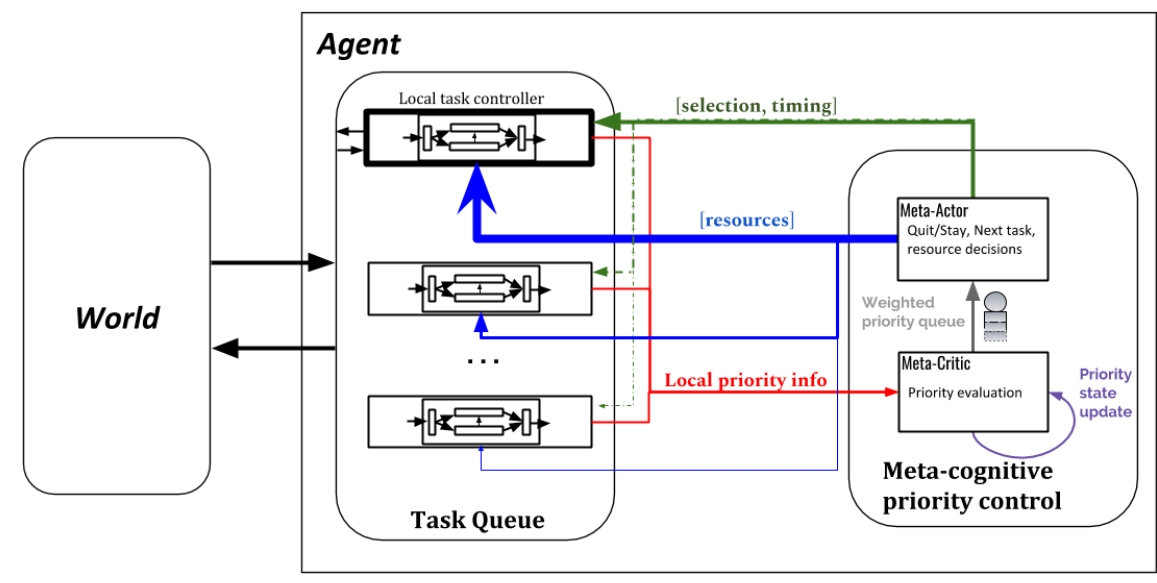

Figure 5: Engagement as the result of a metacognitive priority control system. Different local task actors determine which task goals are driving overt actions (within-task control). Outside is a meta-level controller that performs across-task control, assigning resources and activation to each task controller (e.g., by specifying goals for the task control loop). Local task actors send back local priority information from each task to the meta-critic. The meta-critic computes an overall priority scores, integrating across longer time scales, while the actor computes priority scores across a salient task set ("queue"). The metacognitive system produces action emissions that must be coordinated: disengagement, next task selection, resource allocation. Resources are allocated to each local task actor, as well as a "To go" signal, indicating which actor is active (which determines both disengagement and next task selection).

self-regulation. While self-regulation is often distinct from research on cognitive control, the two are closely related (Hofmann, Schmeichel, \& Baddeley, 2012) in particular in terms of inhibition. Inhibition refers to the disruption of automated processing or prepotent responses by top-down control (Miyake et al., 2000), which can be considered a kind of control resource which is costly (Shenhav, Botvinick, \& Cohen, 2013). Hierarchical control in self-regulation theories provides a similar view of inhibition, in that higher level goal regulation can disrupt currently enacted goal systems (Carver, C. S., \& Scheier, 1998). Certain types of self-regulation is described as costly or requiring some resource that can be depleted. Some of these resources might be similar in type to biophysical, in that an explicit energetic cost might be associated with their use (Feldman \& Barshi, 2007)

\section{Disengagement}

While engagement can fluctuate due to allocation of these energetic and cognitive resources, disengagement requires allocating no resources to the previously allocated task. The decision to disengage is often viewed as the decision to allocate attentional resources somewhere else, or due to a mechanistic failure (Baumeister \& Vohs, 2007). Impulsivity or ego depletion, for example, frame disengagement as a failure of selfregulation; a failure to properly allocate cognitive resources to the given task. An alternative view is that these resources and disengagement covary since they are both set by a task's overall priority. Switching tasks implies quitting the current task, so the decision to disengage in one task implies engagement in another simply due to the existence of multiple tasks, as well as uncertainty of task completion.
Debate over interpretation of the 'marshmallow task' provides an example of the difficulty in interpreting disengagement. While originally used as an indication of limited self-control (Mischel, Ebbesen, \& Zeiss, 1972), recent research has pointed towards alternative environmental or socio-cultural factors that might explain a child's 'impulsivity' (Watts, Duncan, \& Quan, 2018). An example of this alternative view is from (Kidd et al., 2013), where the marshmallow task was prefaced with an initial task that manipulated the children's expectations of the experimenters reliability. Children first completed an art project where the experimenter promised them a larger set of art tools (e.g., crayons), however some of the children were not provide the promised item. The authors found that those children in the reliable experimenter condition waited significantly longer ( 9 minutes more on average) for the larger reward (i.e., extra marshmallows) than those in the unreliable condition. The children in this instance were not simply discounting the future, but rationally deciding that waiting was not worth it due to low certainty.

Appropriately disengaging from certain goals can be beneficial to individual wellbeing, or simply the product of a tradeoff. While self-interruptions when multitasking can be detrimental (e.g., (Rosen et al., 2013)), they may also be due to the priority applied to differing goals. In a dual-goal setting, for example, people prioritize goals depending on their likelihood of completing both goals (Schmidt \& Dolis, 2009). When only one can be completed time should be allocated to that goal. While there are benefits to engaging deeply in one task, including emotional and motivational, people must allocate time to alternative goals. Psychological flow, therefore, is not just determined by the flow-inducing task, but also the 
lack of alternative available tasks that resources and time can be allocated to (Csikszentmihalyi \& Lefevre, 1989).

\section{Scheduling and time allocation}

The choice of engagement concerns which tasks people engage in in addition to how long they engage in each task. While these decisions can be linked, optimal scheduling indicates that they can often be separated out into two decisions: a choice problem (which task to engage in next) and an optimal stopping problem (when to quit the current task) (Gittins, Glazebrook, \& Weber, 2011). Disengagement relates to both.

A core problem foragers face is the decision to quit a task for possible alternatives. When should an animal quit foraging from a patch of food and move to another? The 'patch model' frames foraging as an optimal stopping problem, which results in consideration of a tradeoff between foreground tasks and background tasks, where the overall environment and possible tasks that can be engaged in impact the amount of time in a foreground task. For example, possibility of predation or reproduction will impact time of feeding (Stephens et al., 2007, ch 7).

There is a important analogy to our framework; people forage across task space much like animals forage across patches. We must make a decision of when to quit a task in the same way animals must decide to quit a patch of food. The number of alternative tasks people can engage in is enormous; people can engage in an unlimited number of possible tasks each day. Consider the game player in the introductory example; while features of the game itself influences how long they play, they have to trade off gameplay time with eating, sleeping, working, or other entertainment. The writer explicitly acknowledges this tradeoff by clearing her schedule. In the case of slot machines for gambling, designers will reshape the entire casino (e.g., making exits hard to find and no view of external environment) along with individual slot machines to 'push' a customer to stick to the machines (Schüll \& Library., 2012).

Within economics, engagement is studied via time-use in terms of how it represents aspects of income, labour, and transportation (Jara-Diaz \& Rosales-Salas, 2017). Time budgets are analyzed using decision utility frameworks, which treat time as a global budget that people spend across tasks with static utilities, dealing with lumped weekly or daily time resources. For example, how much time in a day should you spend on work or travel, given the relative values and costs of each? This contrasts with the dynamic nature of value and engagement, which allows for a more general time allocation.

\section{Conclusion}

We provide an integrated metacognitive framework to understand the gradiated nature of task engagement. This framework introduces the idea of a priority calculation, which jointly sets task resources such as attention and energy, as well as task selection and time allocation. This captures what is otherwise disparate phenomena in an unified fashion, explaining engagement's varied features.

\section{References}

Baumeister, R. F., \& Vohs, K. D. (2007, nov). SelfRegulation, Ego Depletion, and Motivation. Social and Personality Psychology Compass, 1(1), 115-128. doi: 10.1111/j.17519004.2007.00001.x

Botvinick, M. M., \& Braver, T. S. (2014). Motivation and cognitive control: From behavior to neural mechanism. Annual Review of Psychology, 66, 82-113. doi: 10.1146/annurev-psych-010814015044

Bouvier, P., Lavou, E., \& Sehaba, K. (2014). Defining engagement and characterizing engaged-behaviors in digital gaming. Simulation \& Gaming, 45(4-5), 491-507. doi: $10.1177 / 1046878114553571$

Boyle, E. A., Connolly, T. M., Hainey, T., \& Boyle, J. M. (2012). Engagement in digital entertainment games: A systematic review. Computers in human behavior, 28(3), 771-780.

Carrier, L. M., Rosen, L. D., Cheever, N. A., \& Lim, A. F. (2015). Causes, effects, and practicalities of everyday multitasking. Developmental Review, 35, 64-78.

Carver, C. S., \& Scheier, M. F. (1998). On the self-regulation of behavior (No. 9). doi: 10.1017/ CBO9781139174794

Csikszentmihalyi, M., \& Lefevre, J. (1989). Optimal Experience in Work and Leisure. Journal of Personality and Social Psychology, 56(5), 815-822. doi: 10.1037/0022-3514.56.5.815

Dickinson, A., \& Balleine, B. (n.d.). The role of learning in the operation of motivational systems. Learning, Motivation and Emotion (Gallistel, C.R., ed.), 3 .

D’Mello, S., Dieterle, E., \& Duckworth, A. (2017). Advanced, analytic, automated (aaa) measurement of engagement during learning. Educational psychologist, 52(2), 104-123.

Eldar, E., Rutledge, R. B., Dolan, R. J., \& Niv, Y. (2016). Mood as Representation of Momentum. Trends in Cognitive Sciences, 20(1), 15-24. Retrieved from http://dx.doi.org/10.1016/j.tics.2015.07.010 doi: 10.1016/j.tics.2015.07.010

Feldman, J., \& Barshi, I. (2007). The Effects of Blood Glucose Levels on Cognitive Performance: A Review of Literature.

Gittins, J., Glazebrook, K., \& Weber, R. (2011). Multi-Armed Bandit Allocation Indices: 2nd Edition. doi: 10.1002/9780470980033

Hartonen, T., \& Alava, M. J. (2013). How important tasks are performed : peer review. Scientific reports, 3, 1-11. doi: 10.1038/srep01679

Hoffman, B., \& Nadelson, L. (2010). Motivational engagement and video gaming: A mixed methods study. Educational Technology Research and Development, 58(3), 245-270.

Hofmann, W., Schmeichel, B. J., \& Baddeley, A. D. (2012). Executive functions and self-regulation. Trends in Cognitive Sciences, 16(3), 174-180. doi: 10.1016/j.tics.2012.01.006

Jara-Diaz, S., \& Rosales-Salas, J. (2017). Beyond transport time: A review of time use modeling. Transportation Research Part A: Policy and Practice, 97, 209-230.

Kane, M. J., Kwapil, T. R., Mcvay, J. C., \& Myin-germeys, I. (2007). For Whom the Mind Wanders, and When. Psychological Science, 18(7), 614-621. doi: 10.1111/j.14679280.2007.01948.X

Kessler, Y., Shencar, Y., \& Meiran, N. (2009, jun). Choosing to switch: spontaneous task switching despite associated behavioral costs. Acta psychologica, 131(2), 120-128. Retrieved from http://www.ncbi.nlm.nih.gov/pubmed/19386295 doi: 10.1016/j.actpsy.2009.03.005

Kidd, C., Palmeri, H., \& Aslin, R. (2013). Rational snacking: Young children's decision-making on the marshmallow task is moderated by beliefs about environmental reliability. Cognition, 126(1), 109-114. doi: 10.1016/j.cognition.2012.08.004

Matthews, G., Warm, J. S., Reinerman-Jones, L. E., Langheim, L. K., Washburn, D. a., \& Tripp, L. (2010). Task engagement, cerebral blood flow velocity, and diagnostic monitoring for sustained attention. Journal of experimental psychology. Applied, 16(2), 187-203. doi: 10.1037/a0019572

Mischel, W., Ebbesen, E. B., \& Zeiss, A. R. (1972). Cognitive and attentional mechanisms in delay of gratification. Journal of personality and social psychology, 21, 204-218. doi: $10.1037 / \mathrm{h} 0032198$ 
Miyake, A., Friedman, N. P., Emerson, M. J., Witzki, A. H., Howerter, A., \& Wager, T. D. (2000). The unity and diversity of executive functions and their contributions to complex "Frontal Lobe" tasks: a latent variable analysis. Cognitive psychology, 41(1), 49-100. doi: 10.1006/cogp.1999.0734

Mobbs, D., Hagan, C. C., Dalgleish, T., Silston, B., \& Prevost, C. (2015). The ecology of human fear: Survival optimization and the nervous system. Frontiers in Neuroscience, 9(FEB), 1-22. doi: 10.3389/fnins.2015.00055

Newell, A., \& Card, S. K. (1985). The prospects for psychological science in human-computer interaction. Human-computer interaction, 1(3), 209-242.

Niv, Y., Joel, D., \& Dayan, P. (2006). A normative perspective on motivation. Trends in cognitive sciences, 10(8), 375-81. doi: 10.1016/j.tics.2006.06.010

Reschly, A. L., \& Christenson, S. L. (2012). Jingle, jangle, and conceptual haziness: Evolution and future directions of the engagement construct. In S. L. Christenson, A. L. Reschly, \& C. Wylie (Eds.), Handbook of research on student engagement (pp. 3-19). Boston, MA: Springer US. doi: 10.1007/978-1-4614-2018-71

Rosen, L. D., Carrier, L. M., \& Cheever, N. A. (2013). Facebook and texting made me do it: Media-induced task-switching while studying. Computers in Human Behavior, 29(3), 948-958.

Ryan, R. M., Rigby, C. S., \& Przybylski, A. (2006, nov). The Motivational Pull of Video Games: A Self-Determination Theory Approach. Motivation and Emotion, 30(4), 344-360. doi: 10.1007/s11031-006-9051-8

Schmidt, A. M., \& Dolis, C. M. (2009). Something's got to give: the effects of dual-goal difficulty, goal progress, and expectancies on resource allocation. The Journal of applied psychology, 94(3), 678-91. doi: 10.1037/a0014945

Schüll, N. D., \& Library., E. (2012). Addiction by design: machine gambling in Las Vegas. doi: 10.2307/j.ctt12f4d0

Shenhav, A., Botvinick, M. M., \& Cohen, J. D. (2013). The expected value of control: an integrative theory of anterior cingulate cortex function. Neuron, 79(2), 217-240.

Sinatra, G. M., Heddy, B. C., \& Lombardi, D. (2015). The challenges of defining and measuring student engagement in science. Taylor \& Francis.

Stephens, D. W., Brown, J. S., \& Ydenberg, R. C. (2007). Foraging: Behavior and Ecology.

Sutton, R. S., \& Barto, A. G. (1998). Reinforcement Learning : An Introduction. doi: 10.1016/j.brainres.2010.09.091

Watts, T. W., Duncan, G. J., \& Quan, H. (2018). Revisiting the Marshmallow Test : A Conceptual Replication Investigating Links Between Early Delay of Gratification and Later Outcomes. doi: $10.1177 / 0956797618761661$

Whitton, N., \& Moseley, A. (2014). Deconstructing engagement: Rethinking involvement in learning. Simulation \& Gaming, 45(45), 433-449. 